\title{
TOURS ET DÉTOURS (VU DE FRANCE)
}

\section{par Franc Schuerewegen}

\author{
"Je ne connais rien de plus servile, de plus \\ méprisable, de plus lâche, de plus borné qu'un \\ terroriste"
}

- Chateaubriand, Mémoires d'outre-tombe

Je le dis avec tristesse car la nouvelle n'est pas bonne : la France va mal. Le pays est en crise, ses maux sont multiples. Les Français, du reste, le savent et s'en plaignent amèrement. On a pu parler à cet égard d'un « nouveau courant de pensée décliniste » auquel l'ouvrage de Nicolas Baverez, La France qui tombe, semble avoir donné le coup d'envoi. Je ne dirai rien ici du livre de Nicolas Baverez qui n'est malheureusement pas un bon livre. C'est dommage, le sujet méritait mieux. ${ }^{1}$ M'intéresse en revanche une série de trois romans parus en France alors même que le phénomène Baverez bat son plein, c'est-àdire en l'automne 2003. Leur sujet : la terrible journée du 11 septembre 2001. La transition est un peu brusque, j'en conviens ; on me reproche de sauter du coq à l'âne, ce n'est pas le cas. D'une certaine manière, nous passons du même au même car les deux plans ou séries sont liés. Je soutiens en effet ici l'hypothèse selon laquelle, en 2003, dans les romans quej'ai choisi d'étudier, la tragédie new-yorkaise apparaît comme une sorte d'excuse discursive, comme un pur prétexte si l'on veut. En clair : cela parle d'Amérique mais c'est de la France qu'il s'agit, de son malaise identitaire, de sa place changeante dans un monde mondialisé. La France qui tombe regarde, fascinée, des tours qui tombent et verse une larme ou deux. Mais pour qui donc sont ces larmes? Tout le problème, comme on va voir, est là. 
Paraissent lors de la rentrée 2003 : Windows on the World de Frédéric Beigbeder, 11 septembre mon amour de Luc Lang et Allah Superstar du journaliste algérien Yassir Benmiloud. Comme leur titre l'indique plus ou moins clairement selon les cas, les trois textes commémorent Nine-eleven sous la forme d'une fiction romanesque. Je précise qu'il y eut d'autres parutions à la même époque ; il en existe de plus récentes aussi, la source ne s'est pas tarie. Sur le drame du 11 septembre, une vaste bibliothèque est en train de se constituer dont il faudrait entreprendre l'inventaire un jour. ${ }^{2}$ Je m'en tiendrai pourtant, dans un premier temps du moins, aux trois titres que je viens de nommer. Ils me permettront, j'espère, d'illustrer quelle est la sorte de quiproquo auquel les événements new-yorkais, dans le roman français, ont pu donner lieu. Parler de l'autre y est une façon détournée de parler de soi. Le roman « américain » devient un exercice d'introspection, voire un lamento parfois outrageusement pathétique. Je dis cela surtout pour Luc Lang mais n'anticipons pas.

\section{Le peintre de la vie moderne}

« Je suis le Céline musulman de ma génération et comme lui je peins la banlieue avec mon pinceau entre les dents » (Allah Superstar 202). Le ton est donné d'emblée, le lecteur sait donc à quoi s'attendre : Yassir Benmiloud raconte Nine-eleven vu de la banlieue française. Son but semble avoir été de produire une sorte de version light ou postmoderne du Voyage au bout de la nuit. Céline est son héros, son prédécesseur, son exemple. Ce que dit aussi la suite du passage que je suis en train de citer :

Comme quoi Céline il était le reflet de son époque et que son écriture elle puisait son encre brune dans la France raciale de l'Exposition coloniale et c'est pour ça que c'était bon parce que c'était vrai, et moi je suis le reflet de mon époque basanée et mon écriture elle puise son pur sang arabe dans la banlieue raciale du même nom. (202) 
Tout cela est un peu troublant, et c'est le moins qu'on puisse dire. Benmiloud, clairement, cherche à faire peur aux bourgeois, ainsi qu'à quelques autres sans doute. Allah Superstar se donne à lire comme un petit monument de la pensée politiquement incorrecte, c'en est d'ailleurs à mes yeux le principal intérêt. Benmiloud va loin, trop loin. Il n'empêche que, malgré les audaces de notre Bardamu « beur », malgré la volonté de provocation qui est constante, l'auteur ne dépasse à aucun moment les limites d'une esthétique traditionnellement réaliste. Le flirt avec la pensée politiquement incorrecte et la volonté de mimésis vont donc de pair. Le roman est un miroir, un « reflet», le terme est dans le texte. Le « Céline musulman de sa génération » est aussi de ce point de vue un Stendhal au petit pied. Un très petit Stendhal, équipé de pieds minuscules, je m'empresse de l'ajouter. Mais je préfère également laisser cet aspect de côté ici vu que je ne cherche pas à juger ce texte, simplement à l'analyser.

Voici l'essentiel en ce qui me concerne. Pour que le roman de Benmiloud puisse fonctionner comme représentation réaliste, j'entends par là comme image ressemblante, comme portrait, l'auteur s'est vu obligé d'ajouter un révélateur, si l'on préfère, un médiateur permettant de faire le lien entre l'image et le modèle. Il m'est d'avis que le médiateur, ici, c'est très exactement la journée du 11 septembre 2001. C'est bien en effet la tragédie américaine, et l'impact qu'elle a eu, qui permet à Benmiloud de mettre en scène de façon convaincante le questionnement identitaire qui est le thème central de son roman. Pour preuve, la réaction du père du narrateur, né à Alger et dont la France est le pays d'accueil, au moment d'apprendre les événements du WTC à la télévision. Le personnage dit ceci : «Je sais pas qui c'est le fils de pute il a fait ça mais il vient manger le couscous chez moi quand il veut » (143). C'est un peu direct certes, mais cela a l'avantage d'être clair. L'Amérique est l'ennemi, celui grâce à qui les deux avions se sont encastrés dans les tours du World Trade Center, un allié. Dans la banlieue française on réagit comme on a réagi entre autres en Palestine au moment de l'attentat. La haine produit la haine, chose bien connue. Il n'empêche que le fils de l'homme qui s'exprime de la sorte, et qui partage les sympathies son père, je me permets d'insister sur ce point, vit en étroite 
symbiose avec ce qu'il faut bien appeler une certaine forme de culture américaine : la musique rap, Al Pacino dans le rôle de Scarface (« moi je crois en Dieu et Al Pacino est son prophète » [97]) ou, encore, la playstation, meuble indispensable dans le type de logement qu'occupe le « jeune d'origine difficile ». L'Amérique est l'ennemi en même temps que l'exemple. Sans doute est-elle l'ennemi parce qu'elle fonctionne simultanément comme exemple. En termes psychologiques : Benmiloud a mis en place une structure de double contrainte ou double bind. La chose que l'on déteste est aussi la chose que l'on aime, et dont on dépend:

\begin{abstract}
Donc je vois que Bala et Abdel sont sur GTA et ils gravissent les échelons de la Mafia comme Tony Montana et justement le jeu il se passe en Floride dans les années 80 et tu as tous les tubes de l'époque en fond sonore genre Kool \& The Gang, Motley Crue. (106)
\end{abstract}

Prénoms arabes, Grand Theft Auto, Tony Montana, Nineeleven. Tout cela est présent à la fois chez Benmiloud dans un troublant pot-pourri qui se veut aussi une sorte de condensé du climat de l'immédiat après 11 septembre dans le milieu en question. Certes, les jeunes de la cité n'ont pas attendu Ben Laden pour se tailler un comportement d'américanophile contradictoire, basé sur une forme de dénégation (Verneinung) au sens freudien, c'est-à-dire sur la figure paradoxale d'une acceptation-rejet. Il n'empêche que Nine-eleven les a pour ainsi dire révélés à eux-mêmes, c'est le miroir où ils se sont reconnus. J'oublie d'ajouter que le narrateur de Benmiloud répond au nom de Kamel Hassani dit « Léon » qui est le prénom français que lui a donné sa mère. Kamel-Léon, caméléon donc, ce qui donne à la fiction un caractère allégorique ou symbolique. Benmiloud raconte l'histoire d'un personnage qui peut prendre toutes les identités possibles parce qu'il n'en a aucune, c'est-à-dire parce qu'il a perdu son identité propre. Si on pousse l'allégorie jusqu'au bout, Kamel Léon est aussi de ce point de vue, malgré son " pur sang arabe », une image du Français. J'entends : le Français de 2003, évoluant dans un univers brassé et métissé et qui, d'après les puristes, les déclinistes, ne serait plus que l'ombre de lui-même. 
Il y aurait d'autres choses à signaler à propos d'Allah Superstar, je me contenterai des quelques remarques que j'ai présentées vu que Beigbeder et Lang attendent en coulisses et qu'on m'interdit d'être long. Disons donc pour en finir un peu précocement avec ce texte que Benmiloud n'est pas l'auteur d'un chef-d'œuvre mais qu'il a su capter une ambiance et prendre le pouls d'une époque. Cela justifie, à mon sens, sa place dans la série que je me suis permis d'ouvrir ici.

\section{Tours de France}

Qu'est-ce que Windows on the World de Frédéric Beigbeder? Je répondrai ici : une fable topographique, l'histoire de deux sites que l'on a voulu mettre en regard. Le narrateur, qui ressemble assez exactement à l'auteur lui-même, entreprend d'écrire, en 2003, à Paris, un livre sur la tragédie américaine de septembre 2001. Pour cela, il se rend quotidiennement au Ciel de Paris, restaurant situé au 56 étage de la tour Montparnasse. C'est là que, tout en prenant le petit déjeuner, il travaille à son roman. On voit que, comme chez Benmiloud, mais d'une autre manière, car nous évoluons évidemment dans un monde différent, la fiction prend ici des allures d'allégorie. Paris a la tour Montparnasse, New York le WTC. Mais si les deux villes de ce point de vue se ressemblent, elles ne sont sûrement pas pareilles. Beigbeder s'en explique dans son récit :

La tour Montparnasse mesure 200 mètres de hauteur. Pour vous faire une idée de la taille du World Trade Center, eh bien vous empilez deux tours Montparnasse l'une sur l'autre et c'est toujours plus petit que le Centre de Commerce Mondial. (43)

On peut parler d'une leçon d'humilité donnée au lecteur français. A côté des Twin Towers, la tour parisienne fait piètre figure. Ridicule copie d'un original majestueux. Or, il en va de même de l'établissement commercial que la tour abrite : 
Le Ciel de Paris est tout ce qui reste du Windows on the World : une idée. Ce concept saugrenu et précieux de restaurant au sommet d'une tour qui domine la ville. Le décor ici est tout noir, avec un plafond imitant un ciel étoilé. Il n'y a pas grand monde ce matin car le temps est maussade. Les gens annulent leur réservation quand il n'y a pas de visibilité. Le Ciel de Paris est noyé dans la brume. (43)

Ici encore on sent clairement le symbole sous l'effet de réel. A New York en 2001, le temps était radieux ; au $107^{\mathrm{e}}$ niveau, il y avait la foule; à Paris, deux ans plus tard, il n'y a plus personne et la ville est plongée dans la brume. On voit donc où vont les sympathies de l'auteur. La tragédie américaine remplit notre writer-in-residence d'un immense sentiment de compassion pour ceux qui en furent les victimes en même temps que d'une sorte de hargne par rapport à la ville où il réside et qu'il déteste. Qu'est-ce que donc la France? Beigbeder répond : le pays de l'esbroufe est des fanfaronnades, des poseurs et des prétentieux. Si Benmiloud ne mâche pas ses mots, Beigbeder a lui aussi l'art de la franchise :

Quant à l'exception culturelle française, contrairement à ce que disait un pédégé viré depuis, elle n'est pas morte : elle consiste à faire des films exceptionnellement chiants, des livres exceptionnellement bâclés, et dans l'ensemble des œuvres d'art exceptionnellement pédantes et satisfaites. (31)

Petit complément ajouté à l'usage du lecteur non initié : le « pédégé viré » est Jean-Marie Messier, businessman français tombé, comme une tour qui tombe. Messier n'est d'ailleurs pas le seul à être tombé de son piédestal, il a entraîné dans sa chute entre autres l'auteur du livre que nous lisons et qui s'inclut dans la liste : « Il va de soi que j'inclus mon travail dans ce triste constat » (31). 
Est-ce de la fausse modestie ? Beigbeder cherche-t-il à s'en tirer par une pirouette facile? Je ne le crois pas. Il me semble plus exact de dire que le propos consiste à être conséquent avec soi-même, le dénigrement anti-français se muant dès lors logiquement en autodénigrement. Tout cela est lié et a la même origine. Tout cela est pareil. Je rappelle que Windows on the World a aussi un côté « autofiction » vu que le roman se donne à lire entre autres comme l'histoire, intime et malheureuse, d'un divorce. Cela aussi doit être pris en compte ici. Problèmes personnels et désarroi de toute une nation marchent de pair. La France va mal ; Frédéric B. a des déboires conjugaux; à New York, les tours tombent.

Si Beigbeder, avec Windows on the World, a donc écrit un roman anti-français, ses mea culpa réitérés n'excluent pas curieusement un chauvinisme presque militant. C'est ce que nous appellerons l'autre côté de la médaille. Il ne peut en effet échapper à l'attention de personne que si le héros-narrateur-écrivain est dans la possibilité d'écrire un livre sur New York, c'est évidemment parce qu'à Paris la tour Montparnasse est toujours debout. Ce contraste est à sa façon crucial. La naine si constamment ridiculisée par Beigbeder, la pâle et insignifiante copie des deux terribles géantes, est ici la condition de possibilité toute matérielle du texte que nous lisons. La tour Montparnasse, minuscule mais intacte, est un lieu de mémoire. Pour l'auteur-personnage, elle est même le seul endroit au monde où il puisse remémorer efficacement les tours gigantesques, sublimes, mais détruites, de New York. En ce sens donc, le roman dit à la fois une chose et son contraire. Un petit réflexe cocardier apparaît à la fin qui vient pour ainsi dire dorer la pilule. Si nous apprenons d'abord que la France qui tombe se mire, douloureusement, narcissiquement, dans la catastrophe new-yorkaise, en fin de compte, c'est son rôle de survivante et de gardienne de la mémoire qui est célébré.

Les conséquences de ce renversement sont nettement perceptibles à la fin où Beigbeder a choisi de présenter le texte de son ultime chapitre en forme de ... Twin Towers. Je rappelle que les pages 368 à 370 de Windows on the World ont été disposées en deux colonnes, 
l'indication « $1028 \mathrm{~h}$ », l'heure où s'est écroulée la tour nord, figurant une sorte d'antenne coiffant l'ensemble (368). Cela a l'air d'une petite spielerei insignifiante, calligramme apollinarien égaré en milieu romanesque ; il s'agit d'un peu plus que cela à mon sens. C'est qu'on peut interpréter doublement l'artifice typographique. Certes, il s'agit tout d'abord d'une figuration ludique en même temps que vaguement spectrale des tours jumelles effondrées, reflecting absences, si l'on peut risquer ici une allusion au projet de Michael Arad. Mais il existe aussi une autre lecture du texte et dont je suis obligé d'ajouter qu'elle a ma préférence : pourquoi n'admettrions-nous pas que les tours en papier de Beigbeder renvoient en vérité à la fois à la tour Montparnasse et au propre livre de l'auteur? pourquoi ne s'agirait-il pas d'une image en abyme de la fiction ainsi que du lieu où la fiction se fabrique ? On rend mieux compte ainsi de la complexité allégorique du texte : Paris est tombée, comme une tour qui tombe, c'en est fini de son hégémonie culturelle et linguistique. Mais le message inverse est également valable et sans doute tout aussi prégnant : à New York, le mondialisme Made in America a pris un coup ; par le projet fou et crapuleux d'une poignée de terroristes, l'orgueil de toute une nation a été cruellement punie. God blesse America. Et Frédéric Beigbeder nous raconte tout cela confortablement installé dans son luxueux et, prétend-il, indestructible observatoire.

\section{These Indians speak French}

Le Nouvel Observateur du 20 octobre 2003 publie un entretien avec Frédéric Beigbeder et Luc Lang à l'occasion de la parution de leurs deux livres. Il s'agit, explique le magazine, de « confronter des points de vue ». L'entretien devient bien vite un pugilat, le désaccord est total. Lang reproche à Beigbeder « qu'il n'est pas possible de faire de la fiction sur un événement de ce type ». Pour lui donc, Nine-eleven relève de l'ineffable. Beigbeder, quant à lui, remarque « que le mot amerloque revient souvent $»$ dans 11 septembre mon amour, ce qu'il interprète comme un signe d'anti-américanisme (58). Le reproche de Lang me paraît peu fondé. Je serais plutôt d'avis, avec Tom Wolfe, que l'auteur de Windows on the World cite en exergue, « qu'un romancier 
qui n'écrit pas des romans réalistes ne comprend rien aux enjeux de l'époque où nous vivons » (10). Autrement dit : pour comprendre un événement comme Nine-eleven, la fiction peut être un tremplin. Cela est vrai en ce qui me concerne. Quant au constat d'anti-américanisme s'agissant de 11 septembre mon amour, il n'est malheureusement que trop fondé. Force est de reconnaître que Luc Lang n'aime pas le peuple américain et qu'il le montre un peu trop, le pire, ou le plus étonnant, étant d'ailleurs que l'auteur lui-même paraît peu conscient de la phobie qui le hante. Il s'agit donc d'une sorte de tache aveugle de son discours.

J'essaie de m'expliquer un peu mieux. Dans l'interview à laquelle je fais allusion, Lang se fait fort que « son livre n'est en rien anti-américain » et qu'il a voulu écrire au contraire « un hommage aux victimes » (58). Je me permets de contredire l'interprétation de l'auteur. Si c'est en effet un processus de victimisation qui obsède Luc Lang, la vraie victime n'est pas celle qu'on pense. Ce n'est pas l'Américain mais le Français ou, si l'on préfère, l'Indien car cela revient ici au même.

Pourquoi ? Il faut entrer pour bien répondre à la question dans le détail du texte. Commençons par le titre qui est un clin d'œil à Marguerite Duras : « Tu n'as rien vu à Hiroshima... J'ai tout vu, tout ». Le dialogue est archiconnu, Lang l'a compris et cherche à le rentabiliser. Mettez 11 septembre à la place d'Hiroshima, vous obtenez un effet touchant. Evidemment, oui... Que l'on ne s'y trompe pas cependant. La référence durassienne ne doit pas faire oublier que l'important n'est pas la thématique visuelle : voir ou ne pas voir Nine-eleven. La chose essentielle est un conflit culturel que le roman, constamment, pointe du doigt sans jamais parvenir à le conceptualiser. C'est ici, comme on va voir, qu' interviennent les Indiens. Luc Lang, auteur, entre autres, d'un livre consacré au sujet éponyme, est un indianophile passionné. ${ }^{3} \mathrm{C}$ 'est d'ailleurs son amour du peuple indien qui est l'origine de son ouvrage le plus récent. Le narrateur de 11 septembre mon amour voyage, en septembre 2003, dans le Montana où il visite une réserve Blackfeet. Si les Indiens le fascinent, c'est qu'ils incarnent à ses yeux une sorte d'humanité primordiale et édénique, un monde d'avant le crime car 
celui-ci ne commence, bien entendu, qu'avec l'arrivée des colons américains. Luc Lang écrit ceci :

Se prouver que cette terre est bien à soi, enfin, à soi-même en toute légitimité, après l'avoir domptée, soumise, après en être mort si souvent, après en avoir éradiqué ceux-les premiers habitants indigènes - qui vivaient là depuis le fond des âges, qui faisaient tellement corps avec cette nature qu'ils en constituaient fatalement la faune, et en avaient justement la sauvagerie animale. (46)

Cela commence gentiment, respectueusement : des Américains sont morts sur le champ d'honneur. Mais la suite est plus dure : l'Américain a aussi donné la mort, et en cela il est coupable. Lang trouve alors son vrai sujet, les invectives d'un indianophile rousseauiste contre les descendants de Buffalo Bill : "Succès total ! Réussite absolue ! 25000 ans d'histoire humaine, au bas mot, reformatés, recalibrés, réordonnés, en moins de 100 ans » (47).

Qu'il y ait eu génocide en Amérique, cela est probablement exact. Les meilleurs historiens ont défendu cette hypothèse que je ne cherche donc pas à contester ici. Mais qu'il faille sombrer à cause de ce constat effectivement peu réjouissant en une sorte de manichéisme enfantin prenant la forme d'une version inversée et ridicule de la parabole hollywoodienne des cow-boys et des Indiens me semble personnellement un peu tiré par les cheveux. J'ai nettement l'impression que dans la vision d'Amérique que présente Luc Lang, un dieu vengeur a voulu punir le peuple américain pour ses crimes en le frappant, dès la fondation de la nation, d'inculture permanente et profonde. C'est ce qui explique ce portrait au vitriol d'un peuple fort certes mais amèrement détesté et superbement méprisé. L'Américain ne sait rien, ne comprend rien, n'à aucun art de vivre. S'il boit dans un gobelet en plastique équipé d'un « bec à lèvre », c'est qu'il n'a jamais quitté le stade du biberon, suggère Lang (209). D'ailleurs, que boit-il ? Du café détestable, entre autres. Quand, par un hasard qui tient du miracle, le Français en voyage, sur 
une aire de parking, se trouve devant « une camionnette à auvent sur laquelle est inscrit Cappuccino and Espresso made in Italy », il jubile littéralement (55). Si le café n'est pas français, il est européen, ce qui, dans la logique du texte, est plus ou moins la même chose. Cela vient de chez nous, donc, c'est bon! Autre exemple, car nous avons à vrai dire l'embarras du choix: le narrateur arrive sur un campus universitaire, après une semaine passé en contexte anglophone, ce qui a été bien dur pour lui, on imagine pourquoi ; là il fait la connaissance d'une enseignante « parlant un français accompli, sans accent » (164). Lang s'abstient de commentaire se contentant de simplement noter ce fait. Mais on imagine sans peine ce que la scène veut dire : un « français accompli », donc, de la culture ! ouf! un instant, on échappe à la barbarie ! Le français, lingua franca ou, plutôt, petite réserve souffreteuse dans un monde dominé. Il y aurait d'autres éléments à signaler, je le répète ; je me contente de ce qui me paraît le plus symptomatique. D'ailleurs, la scène la plus « énorme » dans la perspective qui est la mienne apparaît à la fin où nous retrouvons notre sujet.

Je plante le décor. Nous sommes à Browning, dans le Montana, le 11 septembre 2001, au matin. Dans la maison de Ee-Nees-TooWah-See, Native American Indian, trois personnes sont présentes : l'hôte indien, le narrateur en voyage, et un troisième Français que le texte appelle le « cow-boy ». Le terme est évidemment péjoratif. Le « cow-boy » qui traverse à cheval les Etats-Unis est un traître. C'est sa nation qu'il a trahie, son identité culturelle, son appartenance. Le personnage est habillé en John Wayne. Je parie que s'il s'était coiffé d'un béret basque, Lang l'eût trouvé infiniment plus sympathique. Mais le plus gros scandale est ceci : le John Wayne venu de France refuse de parler sa langue maternelle ; Lang en est tout choqué : « Il refuse de prononcer un seul mot en français, pas même salut ! à mon attention » (240). C'est là la trahison suprême : dire hello à la place de « salut ». Notre petite fable indianophile et américanophobe n'est pas finie. Dans la salle où les trois personnages sont réunis, la télévision est allumée. Nous assistons en direct au drame du 11 septembre. Lang raconte donc ce que tout le monde sait. D'abord, les tours jumelles sont debout resplendissantes au soleil. Puis arrivent les avions, c'est l'impact. A 10h 
05 , heure locale, la première tour s'effondre. Une voix, que le narrateur ne comprend pas, note Lang, crie : « it collapses ! ». Luc Lang insiste sur l'incompréhension qui est effectivement centrale dans la mise en scène qu'il propose :

Je connaissais le collapsus cardio-vasculaire, par exemple, mot latin qui désigne un état pathologique caractérisé par un malaise soudain, intense, une baisse de tension, une accélération du pouls, des sueurs froides, mais en angloaméricain : to collapse, it collapses ! j'ignorais... (238)

Le « j'ignorais » me donne du fil à retordre. A mon avis, un Français un tant soit peu cultivé-le narrateur de Lang l'est-dût-il tout ignorer la langue de Shakespeare, devrait, dans les circonstances données, vu les images qu'il a sous les yeux, et sachant le collapsus latin et français, être capable de déduire de tout cela que « to collapse » signifie probablement quelque chose comme « tomber » dans la langue de Molière. L'auteur de 11 septembre mon amour prétend que ce génie déductif fait défaut à son personnage, je ne le crois pas. Son scénario est truqué. La soi-disant incompétence linguistique de notre chauviniste invétéré voyageant en pays hostile fournit en réalité à l'auteur un prétexte à manipulation symbolique, à allégorisation si l'on veut, car on ne contestera plus que l'allégorie est un procédé essentiel dans les textes qui m'occupent ici. La fin de notre passage, le « collapsus » terminal si on peut dire, confirme ma lecture : puisque le verbe « to collapse » lui est incompréhensible, le narrateur se tourne vers Ee-NeesToo-Wah-See, sollicitant une explication. A son compatriote, au « cowboy » donc, il ne peut évidemment rien demander vu que celui-ci refuse de parler français. Le généreux peau-rouge vient heureusement le tirer d'embarras « mimant de ses deux paumes ouvertes une compression d'air ». Et c'est alors que soudainement tout s'éclaire: « To collapse veut bien dire s'effondrer, oui, je vois d'ailleurs la première tour à droite, qui s'affaisse » (238). 
On a compris quelle est la lecture que pour ma part je propose de la scène : si « it collapses » demeure opaque au voyageur de 11 septembre mon amour, c'est que le « collapsus » auquel il assiste est en vérité le sien propre; plus précisément : c'est que la chose qui tombe, c'est son amour propre, sa fierté de Français cocardier cruellement exacerbée, et contrecarrée, pendant ce bref voyage américain. On observe donc une résistance, et le verbe incompréhensible (qu'est-ce que c'est que ça?) est aussi dans cette optique une figure de l'inacceptable : Non, il n'y a pas eu de "collapsus » de la France, mon pays est toujours grand et puissant. Mais au plus profond de lui-même le personnage sait, comme le sait aussi l'auteur, que l'irrémédiable a d'ores et déjà eu lieu. C'est dès lors une double commémoration que célèbre le roman : en 2003, en France, écrivant son livre, Lang commémore Nine-eleven ; mais la journée de Nineeleven était elle-même, déjà, à ses yeux, la commémoration d'un événement antérieur qui, lui, n'a rien d'américain mais franco-français. Pour dire la même chose d'une autre manière encore : le 11 septembre 2001, Luc Lang, ou son narrateur-je me permets, comme chez Beigbeder, de mettre les deux dans le même sac - a pris conscience de la terrible blessure narcissique que le nouvel ordre mondial a infligée à la France. Mais ce qu'il a entrevu brusquement — « je n'ai rien vu à Hiroshima »-il refuse de l'accepter, le réfutant, le reniant : Verneinung, ici encore. Son américanophobie vient de là, son indianophilie aussi d'ailleurs. La leçon finale et sans doute un peu caricaturale de 11 septembre mon amour est que les Français sont les Indiens d'Europe.

J'arrête ici l'analyse. Comme toujours quand on s'occupe de l'immédiatement contemporain, la chose difficile est de conclure. Je ne prétends pas avoir fait le tour de quoi que ce soit, ce n'était pas mon but. Je me limiterai donc à ces quelques remarques qui sont aussi l'esquisse d'une recherche à venir. Depuis, au moins, l'automne 2003, le 11 septembre est un thème incontournable et, me semble-t-il, nécessaire dans la pensée française contemporaine. Témoin entre autres les romans que nous avons lus. Que ce soit précisément des romans qu'il a fallu placer au centre du propos me paraît d'ailleurs en soi significatif. Philosophes et sociologues se sont eux aussi penchés sur le 
terrible jour de colère du mois de septembre. Que l'on pense entre autres aux analyses de Baudrillard, de Virilio, de Stiegler, de Derrida. ${ }^{4}$ Une étude d'un autre genre, plus ambitieuse que la mienne, n'aurait d'ailleurs aucun mal à montrer que les tours et détours dont usent les romanciers apparaissant aussi dans d'autres types de discours. Le drame du World Trade Center a aussi, force est de l'admettre, fourni aux intellectuels français un défouloir. Si je me suis concentré sur le roman, c'est donc que Benmiloud, Beigbeder, et Lang me semblent en vérité les pionniers d'un genre littéraire à venir et que nous pourrions appeler, si on accepte le néologisme, le roman undeciseptembral. Ce genre m'intéresse non pour des raisons littéraires - est-ce ici un lieu pour parler littérature ?-mais, précisément: sociales, culturelles, psychologiques, et philosophiques. Je retrouve en d'autres mots mon point de départ : voulez-vous tout savoir sur le déclin de la France? ne lisez pas Baverez, lisez Beigbeder, ou Lang, ou Benmiloud ou tel autre : Yann Moix par exemple qui s'amuse à reconstituer dans Partouz, à la manière burlesque et désinvolte qui est la sienne, la vie sentimentale de Mohamed Atta, pilote au service d'Allah. Le roman de Yann Moix est situé dans le milieu échangiste parisien, métaphore, on l'a deviné, des réseaux islamistes. Networking. Nine-eleven est aussi très clairement chez Moix un faux-fuyant ou un déclencheur, le déclic permettant de passer aux aveux. Toute parole a besoin d'un prétexte. La France contemporaine réfléchit sur elle-même quand elle pense à l'Amérique. On le savait déjà. Cela se confirme ìci. Je cite un seul passage du texte de Yann Moix afin de donner une idée de l'ensemble. Il y est question d'un chansonnier populaire, peu importe l'individu, c'est le ton qui compte :

Je n'avais strictement rien contre Francis Cabrel. Seulement voilà : à l'heure où les McDonald's s'implantaient à côté des boîtes à partouz, il aurait été bon que nos artistes, plutôt que de décrire un monde romantique et éthéré qui n'était plus, collassent un tant soit peu à la réalité : or, la France était devenu un pays de partouzeurs. (247) 
La France, pays de partouzeurs. Le lecteur naïf pourrait penser qu'il s'agit d'un hommage à la tradition libertine du XVIIIIe siècle, il n'en est rien. Le partouzeur est un bon à rien, un frustré, risquons l'équation : un Français. Moix pousse le lamento bien loin mais reste dans le droit fil de ses prédécesseurs.

Tout ceci s'explique-t-il par un anti-américanisme congénital dont Philippe Roger et, dans son sillage, Marc Smeets ${ }^{5}$ ont montré que, depuis les Lumières au moins, il est bien ancré dans les mœurs françaises ? C'est possible en effet. La fiction undeciseptembrale est nouvelle et à la fois ancienne, elle appartient à une tradition.

Puisqu'il est question de tradition, je termine sur ce passage de Chateaubriand dans les Mémoires d'outre-tombe. Le mémorialiste, qui fut aussi un grand voyageur, se rappelle Philadelphie, ville dont l'aspect est « monotone ». Suit alors ceci qui me fait frémir :

\begin{abstract}
En général, ce qui manque aux cités protestantes des Etats-Unis, ce sont les grandes œuvres de l'architecture : la Réformation jeune d'âge, qui ne sacrifie point à l'imagination, a rarement élevé ces dômes, ces nefs aériennes, ces tours jumelles dont l'antique religion catholique a couronné l'Europe. Aucun monument, à Philadelphie, à New York, à Boston, ne pyramide au-dessus de la masse des murs et des toits : l'œil est attristé de ce niveau. ${ }^{6}$ (459)
\end{abstract}

La chose qui surprend, et qui me met mal à l'aise, ce sont bien entendu ces « tours jumelles » que Chateaubriand situe en Europe, non en Amérique, pourquoi donc ? Imaginons un instant une planète, un univers, le nôtre, ou presque, où les Twin Towers seraient une spécialité européenne. Horresco referens.

Franc Schuerewegen Université d'Anvers, Université de Nimègue 


\section{NOTES}

${ }^{1}$ Je renvoie au compte rendu de Laurent Mauduit dans Le Monde des livres du 3 octobre 2003 : « Nicolas Baverez établit le diagnostic abrupt d'un pays qui s'enlise » (2003 : VII). J'emprunte au même auteur l'expression « courant décliniste».

${ }^{2}$ Le présent volume veut aussi être une contribution à ce projet.

${ }^{3}$ Les Indiens (2000).

${ }^{4}$ Power inferno (2002), Ville panique. Ailleurs comme ici (2004), Aimer, s'aimer, nous aimer. Du 11 septembre au 21 avril (2003), Le Concept du 11 septembre (2003). Sur ces textes, voir, ici même, les lectures de David Bell et de L. R. Schehr.

${ }^{5}$ Voir sa contribution au volume.

${ }^{6}$ J'étais déjà fermement décidé à me servir de ce passage dans le contexte de mon étude quand je me suis rendu compte que Gérard Genette le signale aussi, le rapprochant d'ailleurs d'une phrase de Toqueville, dans «Chateaubriand et rien» (304). Mais cela se passe en 2002, avant 2003 qui est pour moi l'année de la prise de conscience du «déclin ». Ce qui m'autorise, je crois, à me resservir du même fragment ici.

\section{OUVRAGES CITÉS OU CONSULTÉS}

Baudrillard, Jean. Power Inferno. Requiem pour les Twin Towers. Hypothèses sur le terrorisme. La violence du mondial. Paris : Galilée, 2002.

Baverez, Nicolas. La France qui tombe. Paris : Perrin, 2003.

Beigbeder, Frédéric. Windows on the World. Paris : Grasset, 2003.

Benmiloud, Yassir (Y.B.). Allah Superstar. Paris : Grasset, 2003.

Chateaubriand, François-René de. Mémoires d'outre-tombe. Ed. JeanClaude Berchet. Paris : Classiques de Poche, 44,1989. 
Derrida, Jacques. Le " concept » du 11 septembre. Dialogues à New York (octobre-décembre 2001) avec Giovanna Borradori. Paris : Galilée, 2003.

Genette, Gérard. Figures V. Paris : Editions du Seuil, 2002.

Lang, Luc. 11 septembre mon amour. Paris : Stock, 2003. . Les Indiens. Paris : Stock, 1987.

Mauduit, Laurent. « Nicolas Baverez établit le diagnostic abrupt d'un pays qui s'enlise ». Le Monde des livres. 3 octobre 2003.

Moix, Yann. Partouz. Paris : Grasset, 2004.

«Peut-on romancer le 11 septembre ? Un face-à-face entre Frédéric Beigbeder et Luc Lang ». Le Nouvel Observateur, 25 octobre 2003.

Virilio, Paul. Ville panique. Ailleurs commence ici. Paris : Galilée, 2004. 
